\title{
Leukaemia Cutis: Clinical Features and Outcomes of 56 Patients
}

\author{
Hwa Jung YOOK ${ }^{1}$, Joon Ho SON${ }^{1}$, Yeong Ho KIM ${ }^{1}$, Ju Hee HAN ${ }^{1}$, Ji Hyun LEE ${ }^{1}$, Young Min PARK ${ }^{1}$, Nack-Gyun CHUNG ${ }^{2}$, Hee \\ Je KIM ${ }^{3}$ and Chul Hwan BANG ${ }^{1}$ \\ ${ }^{1}$ Department of Dermatology, ${ }^{2}$ Department of Pediatrics and ${ }^{3}$ Division of Hematology, Department of Internal Medicine, Catholic Hematology \\ Hospital, Seoul St. Mary's Hospital, College of Medicine, Catholic University of Korea, Seoul, Korea
}

\begin{abstract}
Leukaemia is a malignant neoplasm of the haematopoietic system. Cutaneous manifestations of leukaemia are called leukaemia cutis, and are regarded as a sign of poorer prognosis and shorter survival time. A single-institution retrospective review was performed of medical records of patients diagnosed with leukaemia cutis in the dermatology department of Seoul St Mary's Hospital between January 2012 and April 2021. Fifty-six cases with cutaneous leukaemic involvement and underlying haematological malignancy were included (40 acute myelogenous leukaemia, 8 acute lymphoblastic leukaemia, 3 chronic myeloid leukaemia, 2 chronic lymphocytic leukaemia, and 3 myelodysplastic syndrome). Male-female ratio 1.9:1, mean age at diagnosis 45.8 years. Plaques $(28 \%)$ and papules $(27 \%)$ were the most common skin lesions, followed by patches (18\%) and nodules (16\%). Mean time from diagnosis of leukaemia to development of leukaemia cutis was 12.3 months. Forty-six patients (84\%) died during the 7-year follow-up; mean time from diagnosis of leukaemia cutis to death was $\mathbf{5 . 4}$ months. The results suggest that leukaemia cutis is associated with poor outcomes in patients with leukaemia. Comprehensive skin examination of these patients may help diagnose leukaemia cutis early, enabling prompt treatment.
\end{abstract}

Key words: leukaemia cutis; prognosis; leukaemia; acute myelogenous leukaemia; acute lymphoblastic leukaemia.

Accepted Dec 15, 2021, Epub ahead of print Jan 28, 2022

Acta Derm Venereol 2022; 102: adv00647.

DOI: $10.2340 / a c t a d v . v 102.1123$

Corr: Chul Hwan Bang, Department of Dermatology, Seoul St Mary's Hospital, College of Medicine, The Catholic University of Korea, 222 Banpodaero, Seocho-gu, Seoul 06591, Korea. E-mail: mrbangga@naver.com

$\mathrm{L}$ eukaemia is a life-threatening malignant neoplasm of the haematopoietic system $(1,2)$. After a generalization phase in bone marrow and the appearance of leukaemic cells in the peripheral blood, extramedullary manifestations can occur in any organs of the body, including the skin (2). Findings in the skin of leukaemia patients are common and varied, such as nodular lesions, plaques, hyperpigmentation $(3,4)$ or vasculitis (5). Non-specific cutaneous manifestations are mostly associated with abnormal haematopoiesis, such as thrombocytopenic purpura, incompetent granulocytopoiesis causing opportunistic infection including herpes zoster or fungal infection, and cutaneous paraneoplastic disease

\section{SIGNIFICANCE}

Leukaemia is a malignant neoplasm of the blood system and skin infiltration of leukaemia are called leukaemia cutis, regarded as a sign of a poor survival outcome. We performed a retrospective review of 56 patients diagnosed with leukaemia cutis. The mean interval from diagnosis of leukaemia to the development of leukaemia cutis was 12.3 months. Forty-six patients ( $84 \%$ ) died after diagnosis with leukaemia cutis and the mean interval between the diagnosis with leukaemia cutis and death was 5.4 months. Comprehensive skin examination of leukaemia patients may help to diagnose leukaemia cutis early enough and treatment can be applied promptly.

including pyoderma gangrenosum and insect-sting-like skin changes $(2,6,7)$.

Specific cutaneous manifestations of leukaemia resulting from the invasion of neoplastic leukaemic cells into the epidermis, dermis, or subcutis are called leukaemia cutis $(\mathrm{LC})(3,8)$. These manifestations can also be referred to as cutaneous myeloid sarcoma, a rare extramedullary tumour of malignant myeloid cells $(9,10)$. LC can present anywhere on the skin, with variable morphological features including macules, papules, plaques, nodules, and ulcers, which makes it difficult to clinically distinguish from other non-specific skin lesions $(2,11)$. The lesions may be localized or generalized, and the histological features of LC vary with the type of leukaemia (12). The frequency was previously reported to be $2.1-30 \%$ depending on the type of leukaemia and LC can precede leukaemia by several months or years (3). Thus far, the presence of skin infiltration of leukaemia is regarded as a sign of a poorer prognosis and a shorter survival time (13). Despite the importance of the disease, epidemiological data of the incidences and prevalence of LC are relatively lacking. The aim of this study was to report our experiences with 56 patients diagnosed with LC and contribute to the recent knowledge of LC.

\section{METHODS}

A single-institution retrospective review was performed of the medical records of patients diagnosed with LC in the dermatology department of Seoul St Mary's Hospital between the dates of January 2012 and April 2021. Fifty-six cases, which were histologically confirmed to have cutaneous leukaemic involvement with underlying haematological malignancy, were included in 
Table I. Age and sex distribution of leukaemia cutis (LC) patients $(n=56)$

\begin{tabular}{llllll}
\hline & AML & ALL & CML & CLL & Total \\
\hline Total, $n$ & 40 & 8 & 3 & 2 & 3 \\
Male, $n$ & 22 & 8 & 3 & 1 & 3 \\
Female, $n$ & 18 & 0 & 0 & 1 & 0 \\
Age, years, mean \pm SD (range) & $47.9 \pm 15.6(15-72)$ & $30.9 \pm 18.0(1-48)$ & $60 \pm 14.4(44-72)$ & $66.5 \pm 10.6(59-74)$ & $30.3 \pm 25.0(6-56)$ \\
Patients less than 20 years old, $n(\%)$ & $3(8)$ & $3(38)$ & 0 & 19 & \\
\hline
\end{tabular}

AML: acute myelogenous leukaemia; ALL: acute lymphoblastic leukaemia; CML: chronic myeloid leukaemia; CLL: chronic lymphocytic leukaemia; MDS: myelodysplastic syndrome; SD: standard deviation.

the study. The underlying haematological malignancy of patients was classified based on the results of bone marrow biopsies and peripheral blood analysis. The patients' characteristics, clinical features, and prognosis were evaluated. Cases of leukaemia were further classified as acute myelogenous leukaemia (AML), acute lymphoblastic leukaemia (ALL), chronic myeloid leukaemia (CML), chronic lymphocytic leukaemia (CLL), and myelodysplastic syndrome (MDS). French American-British (FAB) classification was not used because of the recent trend of classifying leukaemia according to the World Health Organization (WHO) classification. In the present study, the classification of leukaemia was determined in consultation with the haematology department of Seoul St Mary's Hospital.

The study protocol was approved by the institutional review board of Seoul St Mary's Hospital, The Catholic University of Korea (IRB number: KC20RASI0854). Informed consent was exempted by the board due to the retrospective nature of the study.

\section{RESULTS}

\section{Demographics}

The 56 cases comprised 40 patients with AML, 8 with ALL, 3 with CML, 2 with CLL, and 3 with MDS. The male:female ratio was $1.9: 1$. The mean age at diagnosis was 45.8 years. The mean ages at diagnosis in patients with MDS, ALL and AML were 30.3, 30.9 and 47.9 years, respectively, which was relatively younger compared with that of the other groups. The mean ages at diagnosis in patients with CML and CLL were 60 and 66.5 years, respectively, showing an older age at diagnosis than those with other types of LC (Table I). The number of patients under the age of 20 years was $7(13 \%)$, including 3 patients with AML (8\% of total patients with AML), 3 with ALL (38\% of total patients with ALL), and 1 with $\operatorname{MDS}$ (33\% of total patients with MDS). None of the patients with CML and CLL were under the age of 20 years.

\section{Clinical features and distribution of skin lesions}

The clinical appearance of LC was widely variable. Plaques (28\%) and papules (27\%) were the most common types of skin lesions, followed by patches $(18 \%)$ and nodules $(16 \%)$. There was only one case of LC that appeared as an ulcer (Figs 1 and 2). Most of the skin lesions were asymptomatic $(75 \%, 42 / 56)$, while they were painful in 9 cases $(16 \%)$ and pruritic in 5 cases (9\%). The trunk (35\%) and extremities (28\%) were the most frequent locations of LC. LC lesions occurred less on the scalp (11\%), face (11\%), neck (7\%), and genitalia (3\%). Most lesions were multiple (87\%, 49/56), while 7 cases $(13 \%)$ were solitary. LC that appeared as a systemic eruption occurred in 3 cases $(3 \%)$.

No significant differences in the clinical appearance of LC were found depending on the types of leukaemia. Plaques (32\%) and papules (28\%) were the most com-

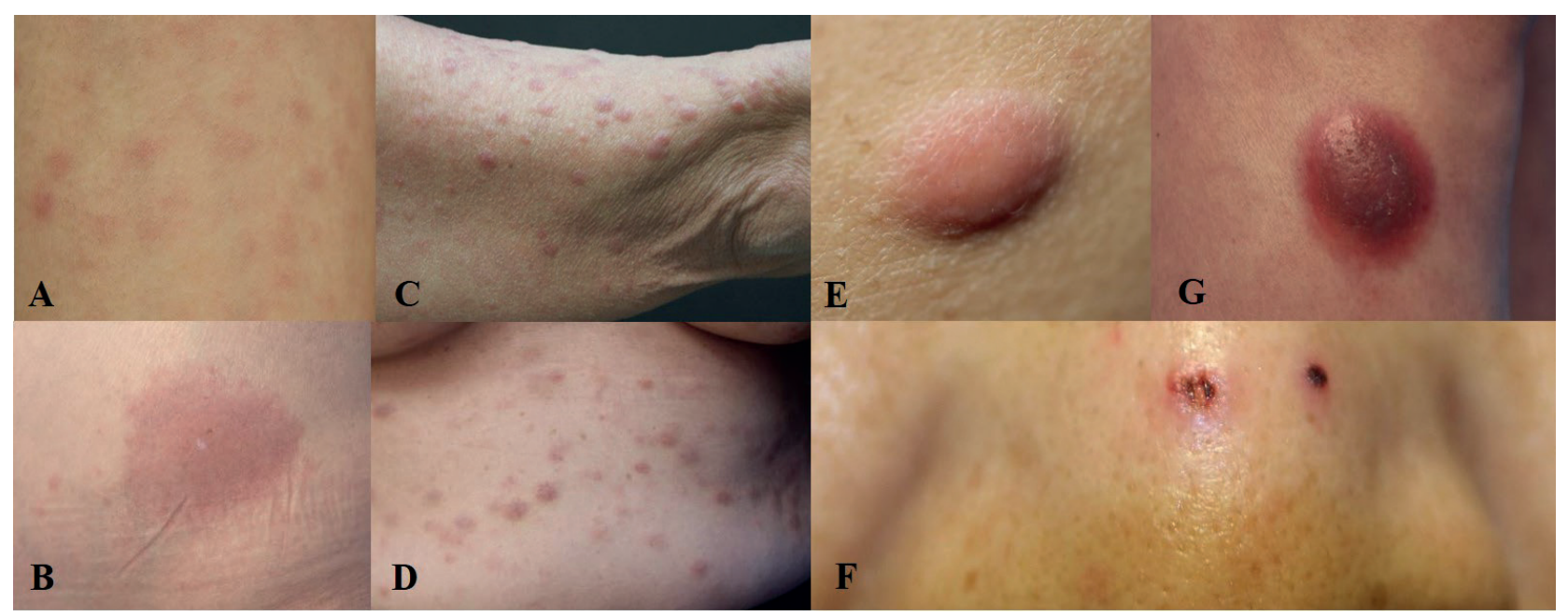

Fig. 1. Clinical appearance of leukaemia cutis (LC). (A) Multiple macules on the abdomen of an acute myelogenous leukaemia (AML) patient. (B) Large patch and satellite macules on the abdomen of an AML patient. (C) Multiple papules on the right arm of an AML patient. (D) Variable-sized plaques on the abdomen of an AML patient. (E) Solitary nodule on the flank area of an AML patient. (F) Ulcer and crust on the nose of a myelodysplastic syndrome patient. (G) Firm nodule on the right lower leg of an AML patient. 


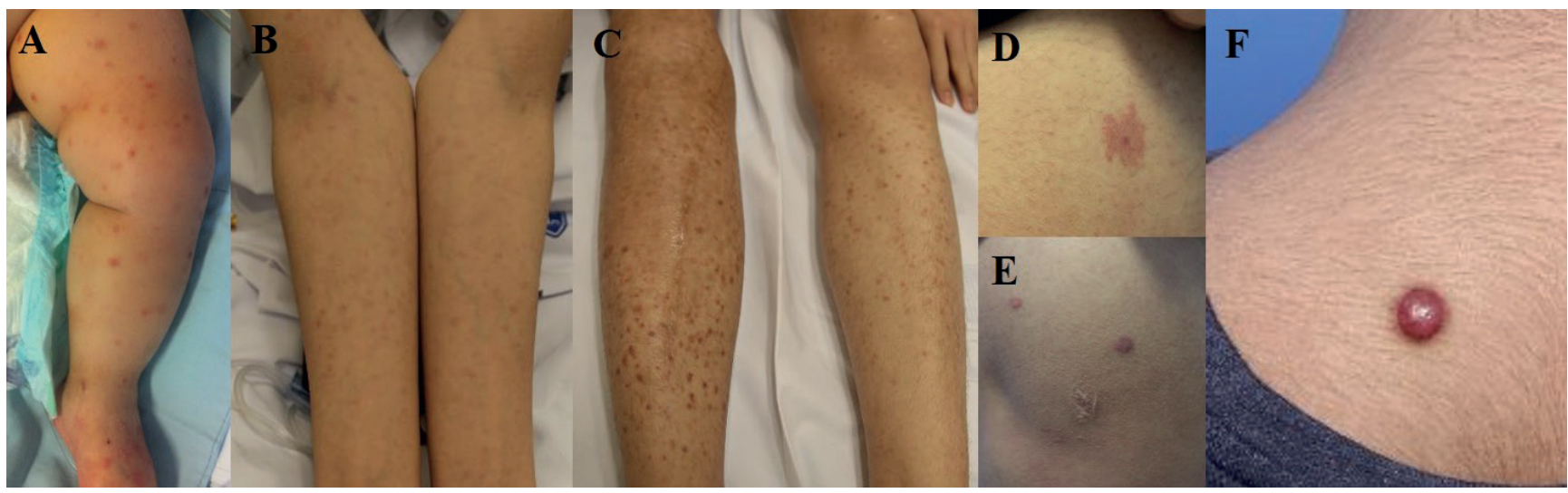

Fig. 2. Gross skin lesions of paediatric leukaemia cutis (LC) patients. (A) Scattered, erythematous papules on the leg of a 1-year-old boy with acute lymphoblastic leukaemia (ALL). (B, C) Numerous patches and papules on the extremities of an 18-year-old boy with acute myelogenous leukaemia (AML). (D, E) Purpuric patch and papules on a 14-year-old boy with ALL. (F) Solitary firm nodule on the upper back of a 15-year-old boy with AML.

mon types of skin lesions in AML, which were mainly distributed on the trunk (37\%) and extremities (33\%). The most common skin lesions in ALL were patches $(28 \%)$, followed by plaques $(22 \%)$ and papules $(22 \%)$, which were mainly distributed on the trunk (42\%). The most frequent locations of $\mathrm{LC}$ in CML were the scalp $(25 \%)$ and face $(25 \%)$. However, patients with LC on the scalp and face also presented LC on other parts of body (Table II; Figs 3 and 4).

\section{Interval from diagnosis of leukaemia and development of leukaemia cutis}

In 47 of 56 patients (84\%), LC developed after the diagnosis of leukaemia. The mean interval from diagnosis of leukaemia to the development of LC was 12.3 months. Separated by type of leukaemia, the mean interval from

Table II. Clinical features and distribution of skin lesions

\begin{tabular}{|c|c|c|c|c|c|c|}
\hline & $\begin{array}{l}\text { AML } \\
n=40 \\
n(\%)\end{array}$ & $\begin{array}{l}\text { ALL } \\
n=8 \\
n(\%)\end{array}$ & $\begin{array}{l}C M L \\
n=3 \\
n(\%)\end{array}$ & $\begin{array}{l}\text { CLL } \\
n=2 \\
n(\%)\end{array}$ & $\begin{array}{l}\text { MDS } \\
n=3 \\
n(\%)\end{array}$ & $\begin{array}{l}\text { Total } \\
n=56 \\
n(\%)\end{array}$ \\
\hline \multicolumn{7}{|c|}{ Clinical appearance } \\
\hline Macule & $6(7)$ & $3(17)$ & $1(12.5)$ & $1(33)$ & $1(14)$ & $12(10)$ \\
\hline Patch & $13(16)$ & $5(28)$ & $1(12.5)$ & $1(33)$ & $1(14)$ & $21(18)$ \\
\hline Papule & $23(28)$ & $4(22)$ & $3(37.5)$ & 0 & $2(29)$ & $32(27)$ \\
\hline Plaque & $26(32)$ & $4(22)$ & $2(25)$ & 0 & $1(14)$ & $33(28)$ \\
\hline Nodule & $14(17)$ & $2(11)$ & $1(12.5)$ & $1(33)$ & $1(14)$ & 19 (16) \\
\hline Ulcer & 0 & 0 & 0 & 0 & $1(14)$ & $1(1)$ \\
\hline \multicolumn{7}{|l|}{ Distribution } \\
\hline Scalp & $6(9)$ & $2(17)$ & $2(25)$ & 0 & $1(17)$ & $11(11)$ \\
\hline Face & $6(9)$ & $2(17)$ & $2(25)$ & 0 & $1(17)$ & $11(11)$ \\
\hline Neck & $5(7)$ & 0 & $1(13)$ & $1(25)$ & 0 & $7(7)$ \\
\hline Trunk & $25(37)$ & $5(42)$ & $1(13)$ & $1(25)$ & $2(33)$ & $35(35)$ \\
\hline Extremity & $22(33)$ & $2(17)$ & $1(13)$ & $2(50)$ & $1(17)$ & $28(28)$ \\
\hline Genitalia & $1(1)$ & 0 & $1(13)$ & 0 & $1(17)$ & $3(3)$ \\
\hline Whole body & $2(3)$ & $1(8)$ & 0 & 0 & 0 & $3(3)$ \\
\hline \multicolumn{7}{|l|}{ Number of lesions } \\
\hline Solitary & $6(15)$ & $1(13)$ & 0 & 0 & 0 & $7(13)$ \\
\hline Multiple & $34(85)$ & $7(87)$ & $3(100)$ & $2(100)$ & $3(100)$ & 49 (87) \\
\hline \multicolumn{7}{|l|}{ Symptoms } \\
\hline Asymptomatic & $30(75)$ & $6(75)$ & $2(67)$ & $1(50)$ & $3(100)$ & $42(75)$ \\
\hline Pain & $6(15)$ & $2(25)$ & $1(33)$ & 0 & 0 & $9(16)$ \\
\hline Pruritus & $4(10)$ & 0 & 0 & $1(50)$ & 0 & $5(9)$ \\
\hline
\end{tabular}

AML: acute myelogenous leukaemia; ALL: acute lymphoblastic leukaemia; CML: chronic myeloid leukaemia; CLL: chronic lymphocytic leukaemia; MDS: myelodysplastic syndrome. diagnosis of leukaemia to the development of LC was 11 months with AML, 23.4 months with ALL, 8.6 months with CML, 5.3 months with CLL, and 11.7 months with MDS. It should be noted that the mean interval from diagnosis with leukaemia to the development of LC in patients with ALL was more than twice the interval in patients with the other types of leukaemia. One patient had skin lesions before the diagnosis of systemic leukaemia, with an interval of 1 month. Eight patients (14\%) had concurrent involvement (Table III). Of the patients with AML, 9 (22.5\%) were post-haematopoietic stem cell transplantation (HSCT) and $11(27.5 \%)$ were refractory to chemotherapy. Among the patients with ALL, 4 $(50 \%)$ were post-HSCT and $1(12.5 \%)$ was refractory to chemotherapy.

In cases of 7 paediatric patients, 2 (29\%) presented $\mathrm{LC}$ at the time of diagnosis of leukaemia. Five patients (71\%) developed LC after the diagnosis of leukaemia, with a mean interval of 13.9 months.

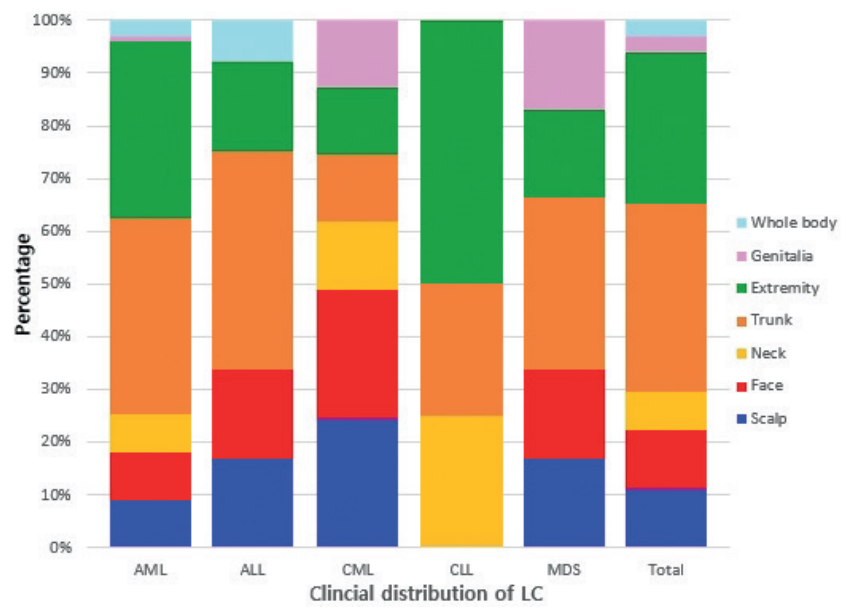

Fig. 3. Bar graph of the clinical features of leukaemia cutis (LC) according to the type of leukaemia. AML: acute myelogenous leukaemia; ALL: acute lymphoblastic leukaemia; CML: chronic myeloid leukaemia; CLL: chronic lymphocytic leukaemia; MDS: myelodysplastic syndrome. 


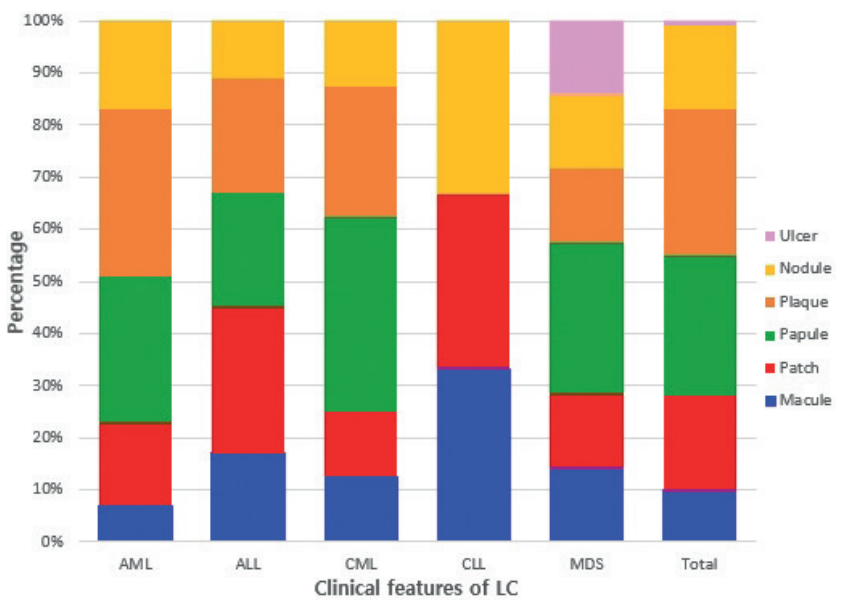

Fig. 4. Bar graph of the clinical distribution of leukaemia cutis (LC) according to the type of leukaemia. AML: acute myelogenous leukaemia; ALL: acute lymphoblastic leukaemia; CML: chronic myeloid leukaemia; CLL: chronic lymphocytic leukaemia; MDS: myelodysplastic syndrome.

\section{Clinical and survival outcomes with leukaemia cutis}

One patient with CML experienced blastic transformation, with $76 \%$ of blast cells in peripheral blood. Among 40 patients with AML, 1 patient with CLL and 3 patients with MDS exhibited transformation into AML. In total, 4 (4/40, 10\%) AML, 4 (4/8, 50\%) ALL, 1 (1/2, 50\%) CLL, and $1(1 / 3,33 \%)$ patient with CML demonstrated internal organ involvement, with bone, liver, spleen or peritoneal space infiltration confirmed on PET/CT.

Forty-six of the 56 total patients $(84 \%)$ died after diagnosis with LC during the follow-up period of 7 years. The mean interval between diagnosis with LC and death was 5.4 months. When classified by types of leukaemia, the mean interval between diagnosis of LC and death was 4.8 months with AML, 9.4 months with ALL, 7.5 months with CML, 3.6 months with CLL, and 9.5 months with MDS (Table IV). Five patients out of the 7 total paediatric patients (71\%) died during the follow-up period and the mean interval between diagnosis with LC and death was 5.8 months.

\section{DISCUSSION}

Leukaemia is a malignant neoplasm of the haematopoietic system, and LC clinically demonstrates skin infiltration by leukaemia $(3,12,14)$. LC presents as generalized or localized papules, nodules, or masses with
Table IV. Survival outcomes of patients with leukaemia cutis (LC)

\begin{tabular}{lllllll}
\hline & AML & ALL & CML & CLL & MDS & $\begin{array}{l}\text { Total } \\
\mathrm{n}(\%)\end{array}$ \\
\hline Patients, $n(\%)$ & 35 & 7 & 1 & 1 & 2 & $46(84)$ \\
$\begin{array}{c}\text { Duration, months, } \\
\text { mean (range) }\end{array}$ & 4.8 & 9.4 & 7.5 & 3.6 & 9.5 & 5.4 \\
\hline
\end{tabular}

AML: acute myelogenous leukaemia; ALL: acute lymphoblastic leukaemia; CML: chronic myeloid leukaemia; CLL: chronic lymphocytic leukaemia; MDS: myelodysplastic syndrome.

characteristic pathological and immunohistochemical manifestations $(3,12)$.

From 2011 to 2017, the 5-year overall relative survival rates for leukaemia patients in the US population (by type) were 87.2\% (CLL), 69.9\% (ALL), 70.6\% (CML), and $29.5 \%$ (AML) (15). In comparison with these US national statistics, the 5-year survival rates for leukaemia patients in the Korean population from 2008 to 2012 (by type) were 44\% (MDS), 85.5\% (CML), 34.8\% (AML), and $52.4 \%$ (lymphoid leukaemia) $(16,17)$.

In the current study, $84 \%$ of patients died after being diagnosed with LC, and 93\% of them died within 10 months. $\mathrm{Su}$ et al. $(6,8)$ reported that $88 \%$ of patients diagnosed with LC died within 1 year of diagnosis in their studies involving ALL, AML, CLL and other leukaemia patients (9). Another study of 26 patients with LC reported a mean survival of 7.6 months for AML and 9.4 months for CML, regardless of treatment modality (18). Similarly, the mean duration of AML and CML in patients with skin involvement was 4.8 and 7.5 months in our study. Although the sample number of each group was small, mean survival of LC according to subgroup was less than 10 months, consistent with previous reports. In a matched-cohort study in 2019, AML patients with LC were 2.06 times more likely to die due to leukaemia, 1.66 times more likely to die due to all causes, and showed a worse 5 -year survival rate $(8.6 \%$ vs $28.3 \%)$ in comparison with the matched patients with AML without LC (9). Similarly, the median 2-year survival for AML patients with LC was $<10 \%$ compared with $30 \%$ in the absence of LC in other studies (19).

The relative survival rate and mean overall survival (OS) in LC is poor, which may be explained by the fact that LC is frequently accompanied by infiltration into other organs $(11,21)$ and it is associated with transformation into the blastic phase, portending disease progression (22). Osio et al. reported a median OS of $24 \mathrm{MDS}$ patients with skin infiltration from skin diagnosis of 62 months,

Table III. Interval from diagnosis of leukaemia to development of leukaemia cutis (LC)

\begin{tabular}{|c|c|c|c|c|c|c|}
\hline & AML & ALL & CML & CLL & MDS & Total \\
\hline Skin lesions following systemic involvement, $n(\%)$ & 36 & 6 & 3 & 1 & 1 & $47(84)$ \\
\hline Duration, months, mean (range) & $11(0.3-64)$ & $23.4(0.7-38)$ & $8.6(3-17)$ & $5.3(5.3)$ & $11.7(11.7)$ & $12.3(0.3-64)$ \\
\hline Concurrent occurrence, $n(\%)$ & 3 & 2 & & 1 & 2 & $8(14)$ \\
\hline Skin lesions preceding systemic involvement, $n(\%)$ & 1 & & & & & $1(2)$ \\
\hline Duration, months, mean (range) & $1(1)$ & & & & & $1(1)$ \\
\hline
\end{tabular}

AML: acute myelogenous leukaemia; ALL: acute lymphoblastic leukaemia; CML: chronic myeloid leukaemia; CLL: chronic lymphocytic leukaemia; MDS: myelodysplastic syndrome. 
with 5 patients $(5 / 24,20 \%)$ developing AML (20). Some studies have suggested that either MDS or CML can transform into systemic acute leukaemia, and the reported risk of MDS transforming into AML is approximately $30 \%(19,20)$; less commonly, transformation can occur in an isolated body area as myeloid sarcoma (21). That is, LC in MDS and CML may indicate progression to acute leukaemia (22). In the current study, a total of 5 patients with MDS, CML and CLL experienced chronic phase to blast phase conversion, presenting with fever and myalgia close to the onset of LC, hence the association between LC and blast formation should be considered. Osio et al. reported that skin lesions occurred when MDS progressed to AML, with a 2-month OS after the occurrence of skin lesions in $7 \mathrm{LC}$ patients with previous MDS. In the current cohort, the mean overall survival of transformed patients was 2.9 months after the development of the skin lesions, which was shorter than the OS of not transformed patients.

In the current study, the most common clinically observed skin lesions were plaques, papules, and patches, as Kaddu et al. reported that LC presented as various morphological features (18). Similarly, Kang et al. and $\mathrm{Li}$ et al. reported that most of the LC lesions presented as papules, plaques, and nodules in their studies (11, 12). No difference was found in the predominant anatomical sites of LC in this study, in comparison with the results of other studies, which reported a predilection of the extremities and the trunk according to the type of leukaemia $(11,13,14)$. Although oral manifestations occur in leukaemia patients and may present as an initial or relapsing feature of the disease (12), none of the patients in the current study showed oral infiltration of leukaemic cells. Any location on the skin of the body could be involved, including mucosa and genitalia, which highlights the importance of detailed skin examinations in patients with leukaemic diseases.

AML with LC shows an incidence ranging from $5 \%$ to $10 \%$ (23), and LC is also seen in other types of leukaemia with varying frequencies $(3,14)$ of $1 \%$ to $3 \%$ in $\operatorname{ALL}(24)$ and $2 \%$ in CML (25). Comparing that the most common type of leukaemia in western countries is $\operatorname{CLL}(3,26)$, AML is most frequent in Korea $(16,17)$, however, LC is most common in AML worldwide $(3,23)$.

In comparison with a case series of 31 paediatric patients reported by Elena et al. (27) (mean age 26.8 months), the mean age of LC diagnosis among our paediatric patients was notably older. No differences were found in LC morphology or distribution among children with different types of leukaemia compared with previous studies, but the sample sizes included in the current study were very small, hence it is difficult to draw any definitive conclusions $(27,28)$.

LC may present as skin lesions before the onset of leukaemia, it may develop as skin infiltration within several months to years after the onset of leukaemia, or skin lesions and systemic leukaemia may develop simultaneously (12). In the current study, $98 \%$ of LC presented after the diagnosis of leukaemia or appeared as a concurrent involvement. Only $2 \%$ of patients had skin lesions before their diagnosis with systemic leukaemia. According to a review article comparing studies of LC between various countries, $71-100 \%$ of LC appeared at the time of diagnosis of leukaemia or thereafter (29). In 3 case series in Korea, the rate of simultaneous occurrence or diagnosis of leukaemia preceding LC was even higher (82-95\%) (29). Also, skin infiltration by leukaemic cells without blasts in peripheral blood samples, which is referred to as aleukaemic leukaemia cutis, occurred in only $7 \%$ of cases (30), but may antedate the appearance of leukaemic cells in peripheral blood samples by months to years $(4,7)$. Therefore, accurate diagnosis of LC has tremendous prognostic importance especially in these cases, and it underlines the value of a long follow-up period.

This study had the following limitations. First, due to the lack of epidemiological data on the incidences and prevalence of leukaemia patients according to the type of leukaemia, we could not identify the incidences of LC by the type of leukaemia. Secondly, the study recruited a relatively small sample size because patients who were referred to dermatology department or who underwent a biopsy were finally included. Further research with a larger group is needed to analyse the incidences of LC depending on the type of leukaemia.

Complete and comprehensive skin examination is critical in leukaemia patients, since LC lesions can present anywhere on the skin including the mucosa. Given the wide variability in appearance, distribution, symptoms, and stages of leukaemia at presentation, dermatologists and haematologists need to consider LC for any new skin lesions in leukaemia patients and should identify its pathology immediately by performing a skin biopsy. Thorough skin examination of leukaemia patients at any time within the course of their disease may help to diagnose LC early enough that treatment can be applied promptly upon initial diagnosis or upon any change in disease conditions.

\section{ACKNOWLEDGEIMENTS}

This work was supported by the National Research Foundation of Korea (NRF) grant funded by the Korea government (MSIT) (number 2020R1C1C1011296).

\section{REFERENCES}

1. Weedon D. Weedon's skin pathology e-book: expert consultonline and print. Milton, ON, Canada: Elsevier Health Sciences; 2009.

2. Wang S-M, Park S-S, Park S-H, Kim N-Y, Kang DW, Na H-R, et al. Pre-transplant depression decreased overall survival of patients receiving allogeneic hematopoietic stem cell transplantation: a nationwide cohort study. Sci Rep 2020; 
10: $1-10$.

3. Wagner G, Fenchel K, Back W, Schulz A, Sachse MM. Leukemia cutis - epidemiology, clinical presentation, and differential diagnoses. J Dtsch Dermatol Ges 2012; 10: 27-36.

4. Angulo J, Haro R, Gonzalez-Guerra E, Farina MC, Martin L, Requena L. Leukemia cutis presenting as localized cutaneous hyperpigmentation. J Cutan Pathol 2008; 35: 662-665.

5. Paydas S, Zorludemir S. Leukaemia cutis and leukaemic vasculitis. Br J Dermatol 2000; 143: 773-779.

6. Su WP. Clinical, histopathologic, and immunohistochemical correlations in leukemia cutis. Semin Dermatol 1994; 13: 223-230.

7. Grunwald MR, McDonnell MH, Induru R, Gerber JM. Cutaneous manifestations in leukemia patients. Semin Oncol 2016; 43: 359-365.

8. Su WD, Buechner S, Li C-Y. Clinicopathologic correlations in leukemia cutis. J Am Acad Dermatol 1984; 11: 121-128.

9. Wang CX, Pusic I, Anadkat MJ. Association of leukemia cutis with survival in acute myeloid leukemia. JAMA Dermatol 2019; 155: 826-832.

10. Vasconcelos ERA, Bauk AR, Rochael MC. Cutaneous myeloid sarcoma associated with chronic myeloid leukemia. An Bras Dermatol 2017; 92: 50-52.

11. Kang YS, Kim HS, Park HJ, Lee JY, Kim HO, Cho BK, et al. Clinical characteristics of 75 patients with leukemia cutis. J Korean Med Sci 2013; 28: 614-619.

12. Li L, Wang Y, Lian CG, Hu N, Jin H, Liu Y. Clinical and pathological features of myeloid leukemia cutis. An Bras Dermatol 2018; 93: 216-221.

13. Watson KM, Mufti G, Salisbury JR, du Vivier AW, Creamer D. Spectrum of clinical presentation, treatment and prognosis in a series of eight patients with leukaemia cutis. Clin Exp Dermatol 2006; 31: 218-221.

14. Haidari W, Strowd LC. Clinical characterization of leukemia cutis presentation. Cutis 2019; 104: 326-330;E3.

15. Howlader N, Noone AM, Krapcho M, Miller D, Brest A, Yu M, et al. SEER Cancer Statistics Review, 1975-2018, National Cancer Institute. Bethesda, MD.

16. Park HJ, Park EH, Jung KW, Kong HJ, Won YJ, Lee JY, et al. Statistics of hematologic malignancies in Korea: incidence, prevalence and survival rates from 1999 to 2008. Korean J Hematol 2012; 47: 28-38.

17. Park EH, Lee HW, Won YJ, Ju HY, Oh C-M, Ingabire C, et al. OCe. Nationwide statistical analysis of myeloid malignancies in Korea: incidence and survival rate from 1999 to 2012.
Blood Res 2015; 50: 204-217.

18. Kaddu S, Zenahlik P, Beham-Schmid C, Kerl H, Cerroni L. Specific cutaneous infiltrates in patients with myelogenous leukemia: a clinicopathologic study of 26 patients with assessment of diagnostic criteria. J Am Acad Dermatol 1999; 40: $966-978$.

19. Krooks JA, Weatherall AG. Leukemia cutis in acute myeloid leukemia signifies a poor prognosis. Cutis 2018; 102: $266 ; 271 ; 272$.

20. Osio A, Battistella M, Feugeas JP, Cuccuini W, Noguera ME, Petrella T, et al. Myelodysplasia cutis versus leukemia cutis. J Invest Dermatol 2015; 135: 2321-2324.

21. Reap L, Goldman L. Focal blast crisis in concomitant myelodysplastic syndrome and chronic myelogenous leukemia. Leuk Res Rep 2020; 14: 100225.

22. Takeuchi S, Akasaka E, Rokunohe D, Kayo SJ, Matsuzaki Y, Ooshima $\mathrm{H}$, et al. Leukemia cutis is an early sign of blast transformation that may mimic a common skin disease: sycosis-like eruptions in chronic myelomonocytic leukemia. J Dermatol 2010; 37: 916-918.

23. Findakly D, Amar S. A rare case of leukemia cutis as the first presentation of a myelodysplastic syndrome to acute myeloid leukemia transformation. Cureus 2020; 12: e8698.

24. Thomas RM, Harrell JE, Rudnick E, Auerbach J, DeBenedetto $A$, Torres A, et al. Leukemia cutis mimicking tumid lupus as the presenting sign in a case of mixed T/B-cell acute lymphoblastic leukemia. JAAD Case Rep 2020; 6: 598-602.

25. Miller MK, Strauchen JA, Nichols KT, Phelps RG. Concurrent chronic lymphocytic leukemia cutis and acute myelogenous leukemia cutis in a patient with untreated CLL. Am J Dermatopathol 2001; 23: 334-340.

26. Mittal A, Gogia A, Mallick S, Gupta R. Leukemia cutis: a rare initial presentation of chronic lymphocytic leukemia. Indian ] Hematol Blood Transfus 2019; 35: 367-368.

27. Andriescu EC, Coughlin CC, Cheng CE, Prajapati VH, Huang JT, Schmidt BA, et al. Pediatric leukemia cutis: a case series. Pediatr Dermatol 2019; 36: 658-663.

28. Kang YS, Lee JH, Park YM, Lee JY. Eleven-year-old girl with multiple nodules on the trunk. Pediatr Dermatol 2015; 32: 415-416.

29. Chang $\mathrm{YW}$, Lee $\mathrm{CH}$, Tseng $\mathrm{HC}$. Leukemia cutis in a medical center in southern Taiwan: a retrospective study of 42 patients. J Formos Med Assoc 2021; 120: 226-233.

30. Vishalakshi V, Torsekar RG, Shinde S. Aleukemic leukemia cutis. Indian J Dermatol Venereol Leprol 2007; 73: 109-111. 